\title{
Inverse Monte-Carlo and Demon Methods for Effective Polyakov Loop Models of SU(N)-YM
}

\author{
Christian Wozar*, Tobias Kästner, Björn H. Wellegehausen, Andreas Wipf \\ Theoretisch-Physikalisches Institut, Friedrich-Schiller-Universität Jena, Max-Wien-Platz 1, \\ 07743 Jena, Germany \\ E-mail: christian.wozarduni-jena.de
}

\section{Thomas Heinzl}

School of Mathematics and Statistics, University of Plymouth, Drake Circus, Plymouth, PL4 8AA, United Kingdom

\begin{abstract}
We study effective Polyakov loop models for $S U(N)$ Yang-Mills theories at finite temperature. In particular effective models for $S U(3) \mathrm{YM}$ with an additional adjoint Polyakov loop potential are considered. The rich phase structure including a center and anti-center directed phase is reproduced with an effective model utilizing the inverse Monte-Carlo method. The demon method as a possibility to obtain the effective models' couplings is compared to the method of SchwingerDyson equations. Thermalization effects of microcanonical and canonical demon method are analyzed. Finally the elaborate canonical demon method is applied to the finite temperature $S U(4)$ YM phase transition.
\end{abstract}

The XXVI International Symposium on Lattice Field Theory

July 14-19 2008

Williamsburg, Virginia, USA

\section{*Presenter.}




\section{Introduction}

The Svetitsky-Yaffe conjecture [1] states that the Yang-Mills finite temperature transition in dimension $d+1$ is described by an effective spin model in $d$ dimensions with short range interactions. This relationship is analyzed for a $S U(3)$ YM theory with adjoint Polyakov loop potential and the $S U$ (4) YM theory in terms of inverse Monte-Carlo (IMC) methods. Ways to perform IMC are provided by demon methods [2, 3]. Specific thermalization effects of these must be discussed to obtain reliable results. Taking these effects into account we compare the corresponding results to a Schwinger-Dyson approach to IMC [佨.

\section{Effective models for Yang-Mills theories}

We start with the well-known lattice Wilson action

$$
S_{\mathrm{W}}=\beta \sum_{\square}\left(1-\frac{1}{N_{\mathrm{C}}} \operatorname{Re} \operatorname{tr} U_{\square}\right), \quad \beta=\frac{6}{a^{4} g^{2}}
$$

and perform a strong coupling expansion (for small $\beta$ ). Since the resulting 'operators' (Polyakov loop monomials) are dimensionless there is no natural ordering scheme. We therefore use a truncation scheme based on the ordering by powers of $\beta$ which are closely related to the dimension of the corresponding group representations and ordering by the distance across which the Polyakov loops are coupled. In compact form the strong coupling expansion is given by

$$
S_{\mathrm{eff}}=\sum_{r} \sum_{\mathscr{R}_{1} \ldots \mathscr{R}_{r} \ell_{1} \ldots \ell_{r}} c_{\mathscr{R}_{1} \ldots \mathscr{R}_{r}} c_{i=1}^{\ell_{1} \ldots \ell_{r}}(\beta) \prod_{i=1}^{r} S_{\mathscr{R}_{i}, \ell_{i}}=\sum_{i} \lambda_{i} S_{i}
$$

with the basic building blocks

$$
S_{\mathscr{R}, \ell} \equiv \chi_{\mathscr{R}}\left(\mathscr{P}_{\boldsymbol{x}}\right) \chi_{\mathscr{R}}^{*}\left(\mathscr{P}_{\boldsymbol{y}}\right)+\text { c.c. }, \quad \ell \equiv\langle\boldsymbol{x} \boldsymbol{y}\rangle .
$$

Here $r$ counts the number of link operators contributing at each order. The coefficients $c_{\mathscr{R}_{1} \ldots \mathscr{R}_{r}}^{\ell_{1} \ldots \ell_{r}}$ are the couplings between the operators $S_{\mathscr{R}_{i}, \ell_{i}}$ sitting at nearest-neighbor (NN) links $\ell_{i} \equiv\left\langle\boldsymbol{x}_{i}, \boldsymbol{y}_{i}\right\rangle$ in representation $\mathscr{R}_{i}$. The effective action hence describes a network of link operators that are collected into (possibly disconnected) 'polymers' contributing with 'weight' $c_{\mathscr{R}_{1} \ldots \ell_{r}}^{\ell_{1} \ldots \ell_{r}}$. One expects the 'weights' or couplings to decrease as the dimensions of the involved representations and interlink distances increase. In a strong coupling (small $\beta$ ) expansion truncated at $\mathscr{O}\left(\beta^{k N_{\mathrm{t}}}\right)$ one has $r \leq k$ and the additional restriction $\left|\mathscr{R}_{1}\right|+\cdots+\left|\mathscr{R}_{r}\right|<k$ with $|\mathscr{R}| \equiv \sum_{i} p_{i}$ for a given representation $\mathscr{R}$ of $S U(N)$ with Dynkin labels $\left[p_{1}, \ldots, p_{N}\right]$.

\section{Effective models for $\mathrm{SU}(3)$ Yang-Mills}

To lowest order $\mathscr{O}\left(\beta^{N_{\mathrm{t}}}\right)$ one finds the universal effective action

$$
S_{\text {eff }}=c_{10} \sum_{\langle\boldsymbol{x} \boldsymbol{y}\rangle} S_{10,\langle\boldsymbol{x} \boldsymbol{y}\rangle} \equiv \lambda_{1} \sum_{\langle\boldsymbol{x} \boldsymbol{y}\rangle}\left(\mathscr{P}_{\boldsymbol{x}} \mathscr{P}_{\boldsymbol{y}}^{*}+\mathscr{P}_{\boldsymbol{x}}^{*} \mathscr{P}_{\boldsymbol{y}}\right)
$$

Our truncated model to order $\mathscr{O}\left(\beta^{2 N_{\mathrm{t}}}\right)$ and with nearest neighbor interactions reads as

$$
\begin{aligned}
S_{\text {eff }}= & \lambda_{1} \sum_{\langle\boldsymbol{x} \boldsymbol{y}\rangle}\left(\chi_{10}\left(\mathscr{P}_{\boldsymbol{x}}\right) \chi_{01}\left(\mathscr{P}_{\boldsymbol{y}}\right)+\text { c.c. }\right)+\lambda_{2} \sum_{\langle\boldsymbol{x} \boldsymbol{y}\rangle}\left(\chi_{20}\left(\mathscr{P}_{\boldsymbol{x}}\right) \chi_{02}\left(\mathscr{P}_{\boldsymbol{y}}\right)+\text { c.c. }\right) \\
& +\lambda_{3} \sum_{\langle\boldsymbol{x} \boldsymbol{y}\rangle}\left(\chi_{10}\left(\mathscr{P}_{\boldsymbol{x}}\right) \chi_{01}\left(\mathscr{P}_{\boldsymbol{y}}\right)+\text { c.c. }\right)^{2} .
\end{aligned}
$$

For a discussion of effective $S U$ (3) Polyakov loop models see [5, 6]. 


\section{Inverse Monte-Carlo - the basics}

The inverse Monte-Carlo (IMC) method [⿰亻也 allows to determine (effective) actions from given configurations. In our case, these are Polyakov loops obtained from gauge configurations generated with the Wilson action. Via IMC we determine the couplings of truncated effective actions which (ideally) would give rise to the same distribution of Polyakov loop configurations.

The IMC procedure is based on an ansatz for the effective action of the type $S_{\text {eff }}=\sum_{i} \lambda_{i} S_{i}$. Translational invariance of the reduced Haar measure leads to Schwinger-Dyson (SD) equations [7]. They constitute an overdetermined linear system for the effective couplings $\lambda_{i}$ which may be solved by least-square methods (see [8, 9, 10]). A second way to determine the couplings $\lambda_{i}$ is the demon method which was successfully applied to $S U(2) \mathrm{YM}$ in [11].

\section{Microcanonical demon method}

Based on the large volume relation between microcanonical and canonical ensemble in statistical physics additional degrees of freedom ("demons") are used to simulate the effective theory together with the demons at a fixed total energy/action [12]. The demon is used as a "thermometer" to measure the coupling of the corresponding part of the effective action.

To simulate a microcanonical system with action $S[\mathscr{P}]=\sum_{i} \lambda_{i} S_{i}[\mathscr{P}]$ we transform the canonical measure to the microcanonical one,

$$
\rho\left[\mathscr{P}, E_{\mathrm{D}}\right] \propto \exp \left(-\sum_{i} \lambda_{i}\left(S_{i}[\mathscr{P}]+E_{\mathrm{D}}^{i}\right)\right) \quad \longrightarrow \quad \delta\left[S_{i}[\mathscr{P}]+E_{\mathrm{D}}^{i}-E_{\text {total }}^{i}\right] .
$$

Each demons' energy $E_{\mathrm{D}}^{i}$ is distributed according to $\rho\left(E_{\mathrm{D}}^{i}\right) \propto \exp \left(-\lambda_{i} E_{\mathrm{D}}^{i}\right), \lambda_{i}$ depending on $\left\langle S_{i}\right\rangle$. The constraint $E_{\mathrm{D}}^{i} \in\left[-E_{0}^{i}, E_{0}^{i}\right], E_{0}^{i}<\infty$ leads to an invertible relation $\left\langle E_{\mathrm{D}}^{i}\right\rangle=f_{i}\left(\lambda_{i}\right)$. Couplings are obtained via the demon method in the following way:

1. Simulate the microscopic (full YM) system without additional demons.

2. Reduce the system for a chosen configuration to a Polyakov loop configuration.

3. Perform a microcanonical simulation of the reduced Polyakov loop system with coupled demons. As discussed below the thermalization procedure should be handled cautious.

4. The mean energy of the demons is directly related to the couplings of the effective theory.

\section{Tuning the microcanonical demon}

In the microcanonical method one YM configuration is reduced to a Polyakov loop configuration to start the microcanonical run. Therefore the method is highly sensitive to the chosen starting configuration. Thus, the effect of choosing specific (well thermalized) configurations with $\left.S_{i}\right|_{\text {config }}$ in the vicinity of $\left\langle S_{i}\right\rangle_{\mathrm{YM}}$ is analyzed below.

For small $E_{0}^{i}$ thermalization problems of the demon arise due to the small acceptance in the update procedure. If $E_{0}^{i}$ is too large the demon is able to (and generically does) take away much energy from the effective system. Thus, configurations within the microcanonical ensemble become independent of the starting configuration after the reduction step. These problems are circumvented in the following way:

1. Choose a large energy range $\left[-E_{0}^{i}, E_{0}^{i}\right]$ of the order $\mathscr{O}\left(\left|\left\langle S^{i}\right\rangle_{\mathrm{YM}}\right|\right)$.

2. Reduce the YM configuration to the Polyakov loop configuration $C_{0}$.

3. For a few times (10 in our case) perform microcanonical simulations with $C_{0}$ as input configuration in every run. The demons' start energies are given by the expectation value $\left\langle E_{\mathrm{D}}^{i}\right\rangle$ in the preceding run.

4. The final run lasts for the same Monte-Carlo time as the preceding runs and is used to measure $\left\langle E_{\mathrm{D}}^{i}\right\rangle\left(\beta_{i}\right)$. 
Finally the contact between microscopic and microcanonical system is based only on one configuration. Further improvements should be possible by using a canonical demon method with improved "thermal contact" to the microscopic system.

\section{Canonical demon method}

In order to use the full statistics of the microscopic system we apply the following algorithm [13]:

1. Simulate the microscopic system according to $e^{-S_{\mathrm{W}}}$ until thermalization.

2. Perform the reduction of the microscopic system to the effective system.

3. Perform $N_{\text {micro }}$ microcanonical updates of the joined system of effective model and demon energies. These updates do not change the total energy $S^{i}+E_{\mathrm{D}}^{i}$.

4. Freeze the demon system and update the microscopic fields up to a new independent configuration. After that proceed again with step 2 .

To deal with thermalization effects of the demons' energies we begin the measurement after $N_{\text {thermal }}$ microscopic configurations with a suitably chosen $N_{\text {thermal }}$.

\section{Observables for $\mathrm{SU}(3)$}

We discuss the YM theory on a $N_{\mathrm{s}}^{3} \times N_{\mathrm{t}}$-lattice. The Polyakov loop $\mathscr{P}_{x}$ is measured in terms of its lattice average,

$$
P \equiv \frac{1}{V} \sum_{x} \mathscr{P}_{x}, \quad V=N_{\mathrm{s}}^{3}
$$

Since we deal with phases where the traced Polyakov loop is located halfway between the $S U(3)$ center elements we project the value of the traced Polyakov loop onto the nearest $\mathbb{Z}_{3}$-axis and define a rotated Polyakov loop by (see Fig. 1)

$$
P_{\text {rot }}=\left\{\begin{array}{cl}
\operatorname{Re} P & : P \in \mathscr{F} \\
-\frac{1}{2} \operatorname{Re} P+\frac{\sqrt{3}}{2} \operatorname{Im} P & : P \in \mathscr{F}^{\prime} . \\
-\frac{1}{2} \operatorname{Re} P-\frac{\sqrt{3}}{2} \operatorname{Im} P & : P \in \mathscr{F}^{\prime \prime}
\end{array} .\right.
$$

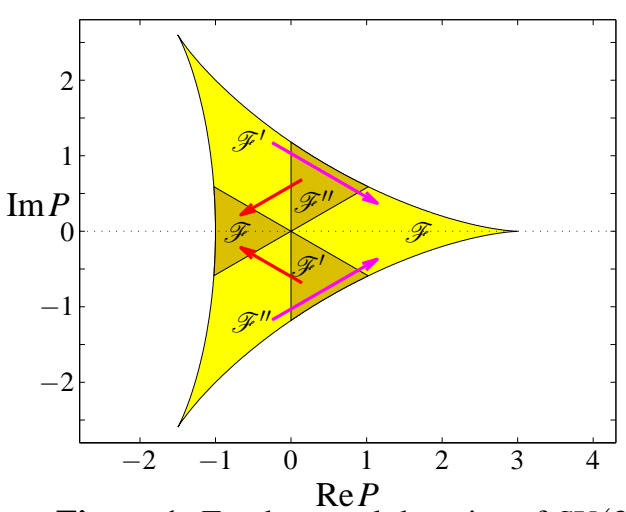

Figure 1: Fundamental domains of $S U(3)$.

\section{The $\mathrm{SU}(3)$ YM phase diagram with adjoint potential}

An adjoint particle with mass $M$ and spin $s$ at temperature $T$ leads to an effective potential [14]

$$
\Delta V_{\text {eff }}=-\left[\frac{(2 s+1) M^{2} T^{2}}{\pi^{2}} K_{2}(M / T) \chi_{11}(\mathscr{P})\right]=T h \chi_{11}(\mathscr{P}), \quad h<0 .
$$

Nevertheless topological excitations allow for a positive $h$ and we therefore study a lattice action

$$
S=\beta \sum_{\square}\left(1-\frac{1}{N_{C}} \operatorname{Re} \operatorname{tr} U_{\square}\right)+H \sum_{x} \chi_{11}\left(\mathscr{P}_{x}\right)
$$

with standard $S U$ (3) Wilson action and adjoint potential with unconstrained parameter $H$.

Simulations of this system near the confinement-deconfinement phase transition on a $12^{3} \times 2$ lattice with varying $\beta$ and $H$ show the phase diagram (Fig. 22) in terms of the rotated Polyakov loop $P_{\text {rot }}$. At $H=0$ the well-known undirected and center-directed Polyakov loop structures related to (de)confinement appear. In the lower half-plane an additional structure arises where the Polyakov loop points into "anti-center" direction.

The additional potential term is already contained in the effective model (2.5). We therefore not only analyze the (de)confinement transition, but also look for a sensible analysis of the anticenter phase. 


\subsection{The confinement-deconfinement transition}

After simulating the (de)confinement phase transition (the upper black curve in Fig. (2) we used IMC with the SD equations as well as the (micro)canonical demon method for deriving couplings of the truncated effective theory. The programming codes were checked by simulating effective theories with fixed couplings and $r e$ producing them consistently with the SD $\left\langle P_{\mathrm{rot}}\right\rangle$ equations and demon methods.

The computed couplings corresponding to one YM coupling $\beta$ are then used to simulate the associated effective theories with a Metropolis algorithm. The resulting expectation values of the rotated Polyakov loop are given in Fig. 自 (left panel). Here the SD equations fail to re-

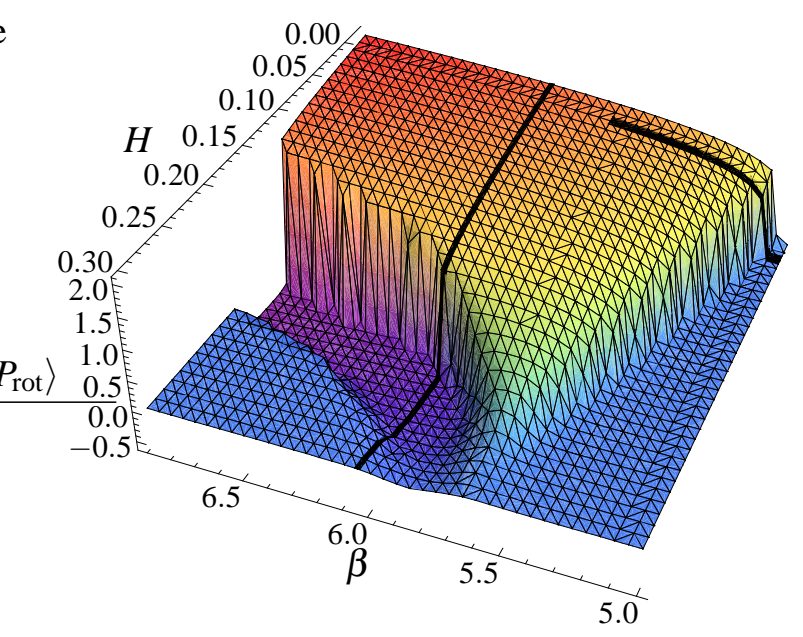

Figure 2: Phase diagram of $S U(3)$ YM with adjoint potential according to Eq. (7.2.). produce the phase transition point whereas the demon methods reproduce $\left\langle P_{r o t}\right\rangle$ near the phase transition showing a better behavior than the SD method in the vicinity of the critical coupling.
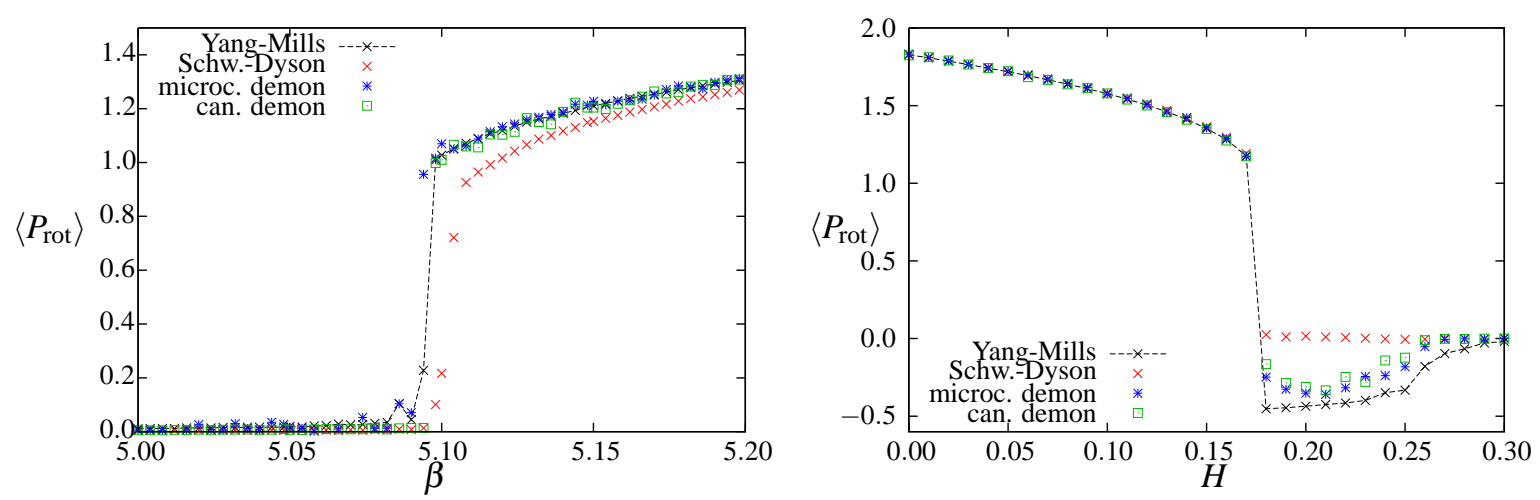

Figure 3: Expectation values of the full theory compared to values produced with effective models after applying IMC methods. Left: (De)confinement transition at $H=0$. Right: Anti-center phase at $\beta=6.05$.

\subsection{The anti-center phase}

At $\beta=6.05$ (vertical black curve in Fig. 2) we analyzed the anti-center phase and compared the resulting expectation values of $P_{\text {rot }}$. Whereas the methods show a quantitative difference in rendering the critical point near the (de)confinement transition the expectation values of $P_{\text {rot }}$ indicate a qualitative difference in the present case (Fig. 3 , right panel). While the SD equations produce a smooth behavior with high accuracy deep in the deconfined phase they fail to reproduce the anticenter phase completely. In contrast demon methods are sensible to the full phase structure. This behavior in the anti-center phase was analyzed with 750 microcanonical runs at $\beta=6.05, H=0.2$ using different randomly chosen starts. The resulting couplings are plotted in the three coupling phase diagram of the effective theory which shows a symmetric, center directed and anti-center directed phase (see Fig. 4 ). Even far away from a phase transition in the microscopic theory the corresponding effective theory can be located in the vicinity of a phase transition of the effective model. 


\section{Using thermalized configurations for the microcanonical demon}

In the microcanonical demon method the starting configuration can be chosen randomly from the full YM ensemble. In contrast we can take configurations with $\left.S_{i}\right|_{\text {config }} \approx\left\langle S_{i}\right\rangle_{\mathrm{YM}}$ ("well thermalized"). In the deconfined phase at $\beta=6.05, H=0$ the projection of the three couplings to the $\lambda_{1}-\lambda_{2}$ plane comparing the different starts is shown in Fig. 5 (left panel).

The extent of the coupling distribution shrinks for the well thermalized configurations compared to the randomly chosen configurations. Additionally the couplings of the well thermalized configurations correspond to almost the same rotated Polyakov loop whereas the couplings derived from the randomly chosen configurations show a much broader distribution of the corresponding rotated Polyakov loops.

\section{Thermalization effects of the canonical demon}

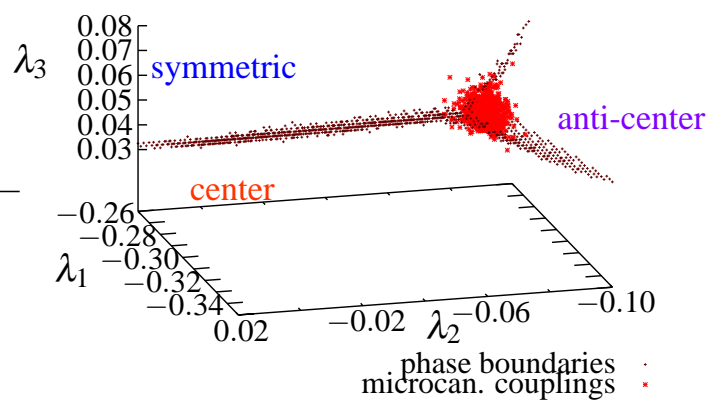

Figure 4: Phase boundaries of the three coupling effective model and couplings derived from the microcanonical method at $\beta=6.05$ and $H=0.2$.

One parameter of the canonical demon method is the number of microcanonical sweeps per microscopic configuration $N_{\text {micro }}$. We analyzed the distribution of the couplings in order to read off any thermalization effects (Fig. 5, right panel). The microcanonical distribution serves as a reference.

Obviously the thermalization of the effective model has to be taken into account. With a small $N_{\text {micro }}$ not enough time is spent to thermalize the effective system completely. The measured couplings refer to a non-equilibrium state of the effective model. Only in the large $N_{\text {micro }}$ limit the couplings describe the thermal equilibrium of the effective model. This behavior is given by the fact that a configuration taken from a thermalized ensemble of the microscopic (YM) system is not necessarily a representative of an equilibrium state of the effective theory [15]. Additionally the couplings obtained via Schwinger-Dyson equations do not correspond to the ones computed with the canonical demon method.
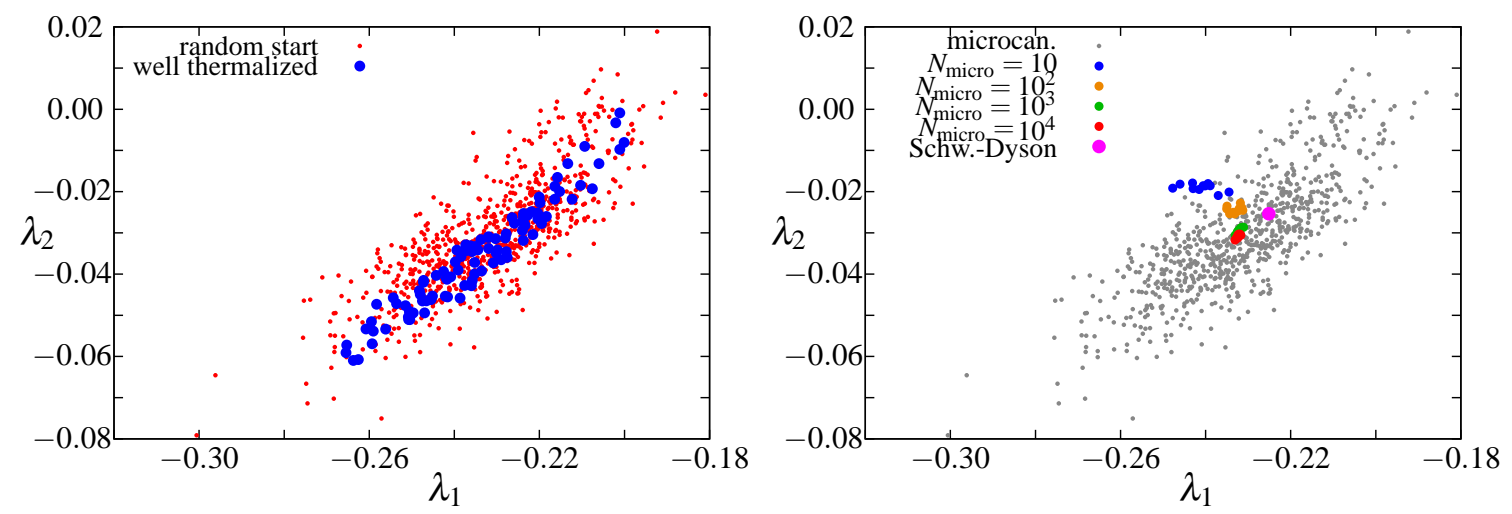

Figure 5: Left: Couplings obtained via the microcanonical demon method with randomly chosen and well thermalized configurations. Right: Thermalization effects due to different $N_{\text {micro }}$ of the canonical demon method.

\section{Outlook to $\mathrm{SU}(4) \mathrm{YM}$}

For the finite temperature phase transition of the $S U(4)$ YM theory on a $6^{3} \times 2$ lattice we applied the IMC method with the canonical demon method. The effective model is a generalization of the three coupling model for $S U(3) \mathrm{YM}$, 


$$
\begin{aligned}
S_{\text {eff }}= & \lambda_{1} \sum_{\langle\boldsymbol{x} \boldsymbol{y}\rangle}\left(\chi_{100}\left(\mathscr{P}_{\boldsymbol{x}}\right) \chi_{001}\left(\mathscr{P}_{\boldsymbol{y}}\right)+\text { c.c. }\right)+\lambda_{2} \sum_{\langle\boldsymbol{x} \boldsymbol{y}\rangle}\left(\chi_{010}\left(\mathscr{P}_{\boldsymbol{x}}\right) \chi_{010}\left(\mathscr{P}_{\boldsymbol{y}}\right)+\text { c.c. }\right) \\
& +\lambda_{3} \sum_{\langle\boldsymbol{x} \boldsymbol{y}\rangle}\left(\chi_{200}\left(\mathscr{P}_{\boldsymbol{x}}\right) \chi_{002}\left(\mathscr{P}_{\boldsymbol{y}}\right)+\text { c.c. }\right)+\lambda_{4} \sum_{\langle\boldsymbol{x} \boldsymbol{y}\rangle}\left(\chi_{100}\left(\mathscr{P}_{\boldsymbol{x}}\right) \chi_{001}\left(\mathscr{P}_{\boldsymbol{y}}\right)+\text { c.c. }\right)^{2} .
\end{aligned}
$$

Even for $S U(4)$ the demon method is a robust way of obtaining couplings of effective Polyakov loop models describing the phase transition (see Fig. 6).

\section{Conclusions}

We studied and compared two ways of obtaining couplings for effective Polyakov loop models which are related to $S U(N)$ YM theories. The application of the inverse Monte-Carlo method with SD equations to the $S U(3)$ YM case leads to stable results only far away from the phase transition. In the vicinity of the phase transition demon methods lead to a much better sampling of expectation values of the Polyakov loop. We tried to reproduce the anti-center phase of a model with standard Wilson action and adjoint Polyakov loop potential by SD and demon methods. The SD method fails to reco-

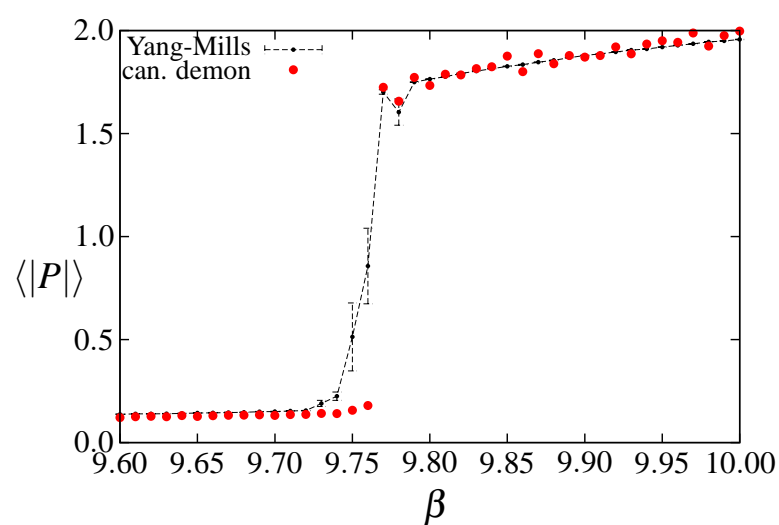

Figure 6: Finite temperature phase transition of $S U(4) \mathrm{YM}$ on a $6^{3} \times 2$ lattice.

ver the phase structure while demon methods are favorable even in this case. The demon method can be generalized straightforwardly to the case of $S U(4)$ YM leading to robust results in the vicinity of the finite temperature phase transition. Combining our experiences with both methods SD equations are less efficient than demon methods near first order phase transitions although SD equations have proven to be very useful for the second order transition in $S U(2)$ YM [8].

When using demon methods much care has to be taken of different thermalization effects. Firstly with microcanonical demons the way of choosing microscopic configurations influences the derived couplings. Secondly when using the canonical demon method thermalization effects (which cannot be cured as discussed in [15]) must be taken into account.

\section{Acknowledgments}

We thank A. Velytsky for an interesting discussion at the conference. TK acknowledges support by the Konrad-Adenauer-Stiftung e.V. and CW by the Studienstiftung des deutschen Volkes. This work has been supported by the DFG grant Wi 777/8-2.

\section{References}

[1] B. Svetitsky and L. G. Yaffe, Nucl. Phys. B 210, 423 (1982).

[2] M. Creutz, A. Gocksch, M. Ogilvie and M. Okawa, Phys. Rev. Lett. 53, 875 (1984).

[3] A. Gocksch and M. Ogilvie, Phys. Rev. Lett. 54, 1772 (1985).

[4] M. Falcioni, G. Martinelli, M. L. Paciello, G. Parisi and B. Taglienti, Nucl. Phys. B 265, 187 (1986).

[5] C. Wozar, T. Kaestner, A. Wipf, T. Heinzl and B. Pozsgay, Phys. Rev. D 74 (2006) 114501.

[6] A. Wipf, T. Kaestner, C. Wozar and T. Heinzl, SIGMA 3 (2007) 006.

[7] S. Uhlmann, R. Meinel and A. Wipf, J. Phys. A 40 (2007) 4367.

[8] T. Heinzl, T. Kaestner and A. Wipf, Phys. Rev. D 72 (2005) 065005.

[9] C. Wozar, T. Kaestner, A. Wipf and T. Heinzl, Phys. Rev. D 76, 085004 (2007).

[10] C. Wozar, T. Kaestner, S. Uhlmann, A. Wipf and T. Heinzl, PoS LAT2007, 341 (2007).

[11] A. Velytsky, arXiv:0805.4450 [hep-lat].

[12] M. Creutz, Phys. Rev. Lett. 50, 1411 (1983).

[13] M. Hasenbusch, K. Pinn and C. Wieczerkowski, Nucl. Phys. Proc. Suppl. 42, 808 (1995).

[14] J. C. Myers and M. C. Ogilvie, Phys. Rev. D 77, 125030 (2008).

[15] E. T. Tomboulis and A. Velytsky, Phys. Rev. D 75, 076002 (2007). 